Article

\title{
Quantum-Granularity Effect in the Formation of Supermixed Solitons in Ring Lattices
}

\author{
Andrea Richaud * (1) and Vittorio Penna \\ Department of Applied Science and Technology and u.d.r. CNISM, Politecnico di Torino, I-10129 Torino, Italy; \\ vittorio.penna@polito.it \\ * Correspondence: andrea.richaud@polito.it; Tel.: +39-011-090-7373
}

Received: 10 December 2019; Accepted: 4 January 2020; Published: 8 January 2020

\begin{abstract}
We investigate a notable class of states peculiar to a bosonic binary mixture featuring repulsive intraspecies and attractive interspecies couplings. We evidence that, for small values of the hopping amplitudes, one can access particular regimes marked by the fact that the interwell boson transfer occurs in a jerky fashion. This property is shown to be responsible for the emergence of a staircase-like structure in the phase diagram of a mixture confined in a ring trimer and to resemble the mechanism of the superfluid-Mott insulator transition strongly. Under certain conditions, in fact, we show that it is possible to interpret the interspecies attraction as an effective chemical potential and the supermixed soliton as an effective particle reservoir. Our investigation is developed both within a fully quantum approach based on the analysis of several quantum indicators and by means of a simple analytical approximation scheme capable of capturing the essential features of this ultraquantum effect.
\end{abstract}

Keywords: Bose-Bose mixtures; mixing; localization; superfluid-Mott transition; quantum phase transitions; entanglement

\section{Introduction}

The possibility to Bose-condense simultaneously two different boson components (whether they are two different chemical elements [1], two different isotopes [2], or two different spin states [3]) and to trap them in optical lattices [4] has opened the door to the investigation of the intriguing phenomenology exhibited by the resulting ultracold mixtures. The behavior of the latter is ruled by the competition among tunneling processes (resulting from the spatial fragmentation of the condensates into separated wells) and intra- and inter-species couplings. Such interplay among different contributions in the overall energy balance of the system results, among the rest, in a rich scenario of mixing-demixing quantum phase transitions [5-9], in the emergence of novel quantum phases [10-12], in the possibility of entangling [13,14] the two bosonic species, and in that of triggering peculiar dynamical regimes [15,16].

In particular, mixing-demixing transitions have been thoroughly described, in the case of small sized lattices, for repulsive [14,17-20] and attractive [21] interspecies couplings. These analyses have highlighted rather complex quantum phase diagrams where various phases, differing in the degree of mixing and localization of the two bosonic species, are recognizable. The latter properties have been shown to be quantifiable by means of suitable indicators originally devised in the context of classical fluids [22], but which can be easily and effectively extended to the case of quantum gases. Mixing-demixing and mixing-supermixing transitions in ultracold bosonic mixtures, which involve the localization of the condensed species in different sites of the lattice, have also been shown to be strongly associated with the presence of criticalities in a number of quantum indicators. The latter include, but are not limited to, the functional dependence of the ground-state energy on model parameters, 
the energy fingerprint (constituted by the structure of the first excited energy levels), and the degree of entanglement between the bosonic species of the mixture [14,18,19,21,23].

In this work, we shine light on a particular aspect of the phenomenology exhibited by two species mixtures confined in optical lattices: the emergence of a quantum-granularity effect resulting from the combination of strong interspecies attractions and weak hopping amplitudes. In these circumstances, in fact, the minority species occupies just one of the available sites and tends to summon the majority species in the same site where it is localized (hence the term "supermixed soliton"). Nevertheless, some bosons of the majority species do not enter the macroscopically occupied lattice site, but remain spread in the remaining ones. The resulting ground-state configuration can be therefore regarded as the union of two parts: the supermixed soliton, which plays the role of a particle reservoir for the majority component, and the remaining sites, which constitute an effective single species system featuring a variable number of particles. In this perspective, the interspecies attraction plays the role of an effective chemical potential, as it can finely control the number of bosons that are injected from (to) the supermixed soliton into (from) the remaining lattice sites. Within this analogy, the jerky interwell transfer of majority bosons occurring in the system is discussed to strongly resemble the well known mechanism underlying the superfluid-Mott insulator transition [24-27]. Recently, there has been considerable interest toward the physics of few body ultracold systems [28] since they allow better understanding the fundamental properties of quantum systems. In the same spirit, a mesoscopic number of particles (instead of a macroscopic one) is employed throughout our analysis to better emphasize the emergence of the quantum-granularity. Moreover, recent experimental advances [29,30] have demonstrated the possibility to realize systems of interacting atoms trapped in ring shaped optical lattices, an achievement that opened the doors to the observation of important phenomena in $1 \mathrm{D}$ physics.

\section{The Two Species Model}

A bosonic binary mixture trapped in a three-well potential (trimer) can be effectively described in terms of the Bose-Hubbard (BH) model. The relevant Hamiltonian,

$$
\begin{gathered}
H=-T_{a} \sum_{j=1}^{3}\left(A_{j+1}^{\dagger} A_{j}+A_{j}^{\dagger} A_{j+1}\right)+\frac{U_{a}}{2} \sum_{j=1}^{3} N_{j}\left(N_{j}-1\right)- \\
-T_{b} \sum_{j=1}^{3}\left(B_{j+1}^{\dagger} B_{j}+B_{j}^{\dagger} B_{j+1}\right)+\frac{U_{b}}{2} \sum_{j=1}^{3} M_{j}\left(M_{j}-1\right)+W \sum_{j=1}^{3} N_{j} M_{j},
\end{gathered}
$$

in fact, can capture the ultraquantum effects originating from the interplay between the spatial fragmentation of the two condensates and the competition among tunneling $\left(T_{a}\right.$ and $\left.T_{b}\right)$ and intra- $\left(U_{a}\right.$ and $\left.U_{b}\right)$ and inter- $(W)$ species couplings [17-21,31]. Operator $A_{j}\left(A_{j}^{\dagger}\right)$ destroys (creates) a species- $a$ boson in the $j$ th site. The same holds for operators $B_{j}$ and $B_{j}^{\dagger}$, which, respectively, destroy and create a species- $b$ boson in the $j$ th site. These operators satisfy standard bosonic commutators: $\left[A_{j}, A_{k}\right]=0$, $\left[A_{j}, A_{k}^{\dagger}\right]=\delta_{j, k},\left[B_{j}, B_{k}\right]=0,\left[B_{j}, B_{k}^{\dagger}\right]=\delta_{j, k},\left[A_{j}, B_{k}\right]=\left[A_{j}^{\dagger}, B_{k}\right]=0$.

Number operators $N_{j}:=A_{j}^{\dagger} A_{j}$ and $M_{j}=B_{j}^{\dagger} B_{j}$ respectively count the number of species- $a$ and species- $b$ bosons in the $j$ th site. Their sums,

$$
\sum_{j=1}^{3} N_{j}=N_{a}, \quad \sum_{j=1}^{3} M_{j}=N_{b},
$$

represent two independent conserved quantities, meaning that $\left[H, N_{a}\right]=\left[H, N_{b}\right]=0$. The system we are going to investigate features a ring geometry, and for this reason, it is understood that $j=4 \equiv 1$ in the summations of Hamiltonian (1). Moreover, in the following, we shall focus on those regimes featuring repulsive intraspecies and attractive interspecies couplings, which means $U_{a}>0, U_{b}>0$ and $W<0$. 


\section{A Continuous-Variable Picture to Investigate the Formation of Supermixed Solitons}

\subsection{The System Phase Diagram}

Bosonic binary mixtures trapped in ring lattices share, irrespective of the number of lattice sites, a rather general mechanism according to which, upon increasing the interspecies attraction $|W|$, the ground-state configuration undergoes deep changes [21]. Basically, the two species are mixed and uniformly distributed in the lattice sites (mixed (M) phase) when $|W|$ is small enough. Conversely, when the latter becomes sufficiently negative, the minority species is localized in one site, while the majority species still occupies all sites, although in a non-uniform way (partially localized (PL) phase). Eventually, further increasing $|W|$, both species are localized in the same site, thus giving place to a state that goes under the name of "supermixed soliton" (supermixed (SM) phase). This scenario is pictorially illustrated in Figure 1.

Phase M

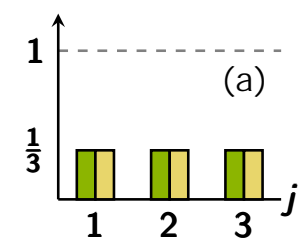

Phase PL

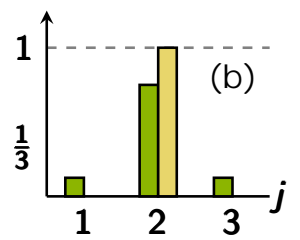

Phase SM

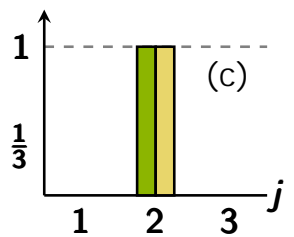

Figure 1. Pictorial representation of some states belonging to phases M, PL, and SM, respectively. Labels 1, 2, 3 correspond to site numbers, while the vertical axis corresponds to (normalized) boson populations $x_{*, j}$ and $y_{*, j}$ characterizing the ground-state configuration. The majority (minority) species is depicted in green (yellow) and corresponds to the left (right) columns of the histograms in each panel. (a) In phase M, the two bosonic species are mixed and uniformly distributed in the ring trimer; (b) in phase PL, the minority species is highly localized, while the majority species occupies all the sites (although in a non-uniform manner); (c) phase SM is characterized by supermixed solitons.

The analytic treatment developed in [21] was based on the continuous variable (CV) picture [32-36], a rather versatile approximation scheme that, under the assumption that the number of particles loaded in the system, $N_{a}$ and $N_{b}$, is large enough, allows one to turn the search for the ground state of Hamiltonian (1) into that for the global minimum of effective potential:

$$
V=\frac{1}{2} \sum_{j=1}^{3} x_{j}^{2}+\frac{\beta^{2}}{2} \sum_{j=1}^{3} y_{j}^{2}+\alpha \beta \sum_{j=1}^{3} x_{j} y_{j},
$$

an expression where variables:

$$
x_{j}:=\frac{N_{j}}{N_{a}}, \quad y_{j}:=\frac{M_{j}}{N_{b}}
$$

represent normalized boson populations and where only two effective parameters,

$$
\alpha=\frac{W}{\sqrt{U_{a} U_{b}}}, \quad \beta=\frac{N_{b}}{N_{a}} \sqrt{\frac{U_{b}}{U_{a}}},
$$

come into play $[19,21]$. It is to be noted, in this regard, that, in the limit of large boson populations, not only the inherently discrete variables $N_{j}$ and $M_{j}$ can be replaced with their continuous counterparts $x_{j}$ and $y_{j}$, but also the contribution of tunneling terms in the potential (2) can be neglected (recall that tunneling energy scales as $N_{c}$, while intra- and inter-species coupling energies scale as $N_{c}^{2}$, where $c=a, b)$. The limit $N_{a} \gg 1, N_{b} \gg 1$, where $N_{b} / N_{a}=$ const, can be regarded as a sort of thermodynamic limit if one resorts to the statistical-mechanical framework discussed in $[37,38]($ see also $[18,19,21])$ and allows one to detect the presence of different phases in the $(\alpha, \beta)$ plane. These 
phases correspond to different classes of ground states of Hamiltonian (1) and differ in the degree of mixing and localization of the two bosonic species, and, at their borders, the energy corresponding to the configuration $(\vec{x}, \vec{y})$, which minimizes (2), regarded as a function of control parameters $\alpha$ and $\beta$, features non-analiticities. More specifically, if the configuration $\left(\vec{x}_{*}, \vec{y}_{*}\right)$ constitutes the global minimum of potential (2), the associated energy,

$$
V_{*}:=V\left(\vec{x}_{*}, \vec{y}_{*}\right):=\min _{(\vec{x}, \vec{y}) \in \mathcal{R}} V(\vec{x}, \vec{y}),
$$

where:

$$
\mathcal{R}=\left\{\left(\vec{x}_{j}, \vec{y}_{j}\right): 0 \leq x_{j}, y_{j} \leq 1, \quad \sum_{j=1}^{3} x_{j}=\sum_{j=1}^{3} y_{j}=1\right\},
$$

features different functional dependences in different regions of the $(\alpha, \beta)$ plane and thus exhibits non-analiticities along the borders thereof. This circumstance strongly resembles the hallmark of quantum phase transitions [39]. Figure 2 illustrates the system phase diagram in the thermodynamic limit (mentioned above), while Table 1 summarizes the ground-state configuration and the associated energy in each of the three phases.

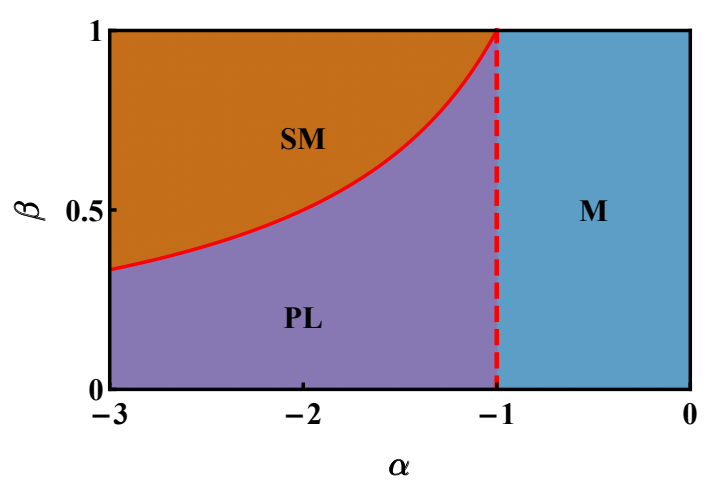

Figure 2. Phase diagram of a (possibly asymmetric) two species bosonic mixture confined in a three-well potential and featuring repulsive intraspecies and attractive interspecies interactions. Each phase is characterized by a specific functional dependence of the energy minimum (5) on effective model parameters (4). Along the red dashed $(\alpha=-1)$ and the red solid $(\beta=-1 / \alpha)$ lines, $V_{*}$ is not analytic, a circumstance that strongly suggests the occurrence of phase transitions. In the former (latter) case, it is the first (second) derivative of $V_{*}$ with respect to control parameter $\alpha$ that is discontinuous.

Table 1. Summary of the typical minimum energy configuration and of the associated energy in

\begin{tabular}{|c|c|c|}
\hline Phase & $\left(\vec{x}_{*}, \vec{y}_{*}\right)$ & $V_{*}$ \\
\hline $\mathbf{M}$ & $\begin{array}{l}x_{*, j}=1 / 3 \quad \forall j \\
y_{*, j}=1 / 3 \quad \forall j\end{array}$ & $V_{*}^{\mathrm{M}}=\frac{1}{6}\left(\beta^{2}+2 \alpha \beta+1\right)$ \\
\hline PL & $\begin{array}{c}x_{*, i}=[1-2 \alpha \beta] / 3 \\
x_{*, j}=[1+\alpha \beta] / 3 \quad \forall j \neq i \\
y_{*, i}=1, \quad y_{*, j}=0 \quad \forall j \neq i\end{array}$ & $V_{*}^{\mathrm{PL}}=\frac{1}{6}\left[1+2 \alpha \beta+\beta^{2}\left(3-2 \alpha^{2}\right)\right]$ \\
\hline SM & $\begin{array}{c}x_{*, i}=1 \\
x_{*, j}=0 \quad \forall j \neq i \\
y_{*, i}=1, \quad y_{*, j}=0 \quad \forall j \neq i\end{array}$ & $V_{*}^{\mathrm{SM}}=\frac{1}{2}\left(\beta^{2}+2 \alpha \beta+1\right)$ \\
\hline
\end{tabular}
each phase. 
To conclude this section, we remark that the presented study encompasses a rather extended portion of the parameters' space. With reference to the definitions (4), in fact, we verified that no additional phases emerge for $\alpha<-3$, while the case of $\alpha>0$ was thoroughly investigated in $[18,19]$. As regards parameter $\beta$, the choice $\beta \in[0,1]$ comes with no loss of generality in that, if $\beta$ happens to be bigger than one, one can always swap species labels and hence come back to the aforementioned interval $\beta \in[0,1]$. Notice also that the asymmetric role of species labels in the definition of $\beta$ (see the formula (4)) implicitly defines a majority species, $a$, and a minority species, $b$.

\subsection{Some Quantum Indicators to Characterize the Different Phases}

In order to better characterize the three possible phases exhibited by the system, one can make use of the "entropy of mixing" and of the "entropy of location", two indicators that are commonly used in physical chemistry $[22,40]$ to quantify the degree of mixing and localization of chemical compounds. In the case of normal fluids, they are defined as:

$$
\begin{gathered}
S_{\text {mix }}(\vec{x}, \vec{y})=-\frac{1}{2} \sum_{j=1}^{L}\left(x_{j} \log \frac{x_{j}}{x_{j}+y_{j}}+y_{j} \log \frac{y_{j}}{x_{j}+y_{j}}\right) \\
S_{l o c}(\vec{x}, \vec{y})=-\sum_{j=1}^{L} \frac{x_{j}+y_{j}}{2} \log \frac{x_{j}+y_{j}}{2} .
\end{gathered}
$$

where $x_{j}$ and $y_{j}$ are the molar fractions of the two compounds in the $j$ th spatial domain and $L$ represents the number thereof (spatial domains result from the discretization of the available volume). As we are dealing with quantum fluids, the system ground state will be, in general, a superposition of different Fock states $|\vec{N}, \vec{M}\rangle$, each one associated with a certain $S_{m i x}$ and $S_{l o c}$, which can be, in turn, determined by means of Formulas (6) and (7) through the mapping (3) (of course, in our case, $L=3$ due to the presence of three sites, which already constitute the most natural way to discretize the system's spatial domain). In this perspective, the quantum version of indicators (6) and (7) reads:

$$
\begin{gathered}
\tilde{S}_{\text {mix }}:=\sum_{\vec{N}, \vec{M}}^{Q}|c(\vec{N}, \vec{M})|^{2} S_{\text {mix }}(\vec{N}, \vec{M}), \\
\tilde{S}_{l o c}:=\sum_{\vec{N}, \vec{M}}^{Q}|c(\vec{N}, \vec{M})|^{2} S_{l o c}(\vec{N}, \vec{M}),
\end{gathered}
$$

where $Q=\frac{\left(N_{a}+2\right) !}{N_{a} ! 2 !} \frac{\left(N_{b}+2\right) !}{N_{b} ! 2 !}$ is the dimension of the Hilbert space of states associated with Hamiltonian (1) and:

$$
c(\vec{N}, \vec{M})=\left\langle\vec{N}, \vec{M} \mid \psi_{0}\right\rangle
$$

is the projection of the ground state $\left|\psi_{0}\right\rangle$ onto Fock state $|\vec{N}, \vec{M}\rangle=\left|N_{1}, N_{2}, N_{3}, M_{1}, M_{2}, M_{3}\right\rangle$.

Other quantum indicators that can be used to detect the presence of different phases [39] in the $(\alpha, \beta)$ plane are the ground-state energy:

$$
E_{0}=\left\langle\psi_{0}|H| \psi_{0}\right\rangle
$$

and the first excited levels:

$$
E_{i}=\left\langle\psi_{i}|H| \psi_{i}\right\rangle .
$$

which indeed constitute a sort of energy fingerprint for quantum phases.

Eventually, in order to evaluate the degree of quantum correlation between the two species, one can introduce the entanglement entropy $(E E)$ relevant to a bipartition of the system space of states in 
terms of species- $a$ and species- $b$ bosons $[17,18,23]$. More specifically, the entanglement between the two quantum fluids reads:

$$
E E=-\operatorname{Tr}_{a}\left(\hat{\rho}_{a} \log _{2} \hat{\rho}_{a}\right),
$$

a formula representing the von Neumann entropy of the reduced density matrix $\hat{\rho}_{a}=\operatorname{Tr}_{b}\left(\hat{\rho}_{0}\right)$ obtained, in turn, by tracing out the degrees of freedom of species- $b$ bosons from the ground-state density matrix $\hat{\rho}_{0}=\left|\psi_{0}\right\rangle\left\langle\psi_{0}\right|$.

Figure 3 illustrates the behavior of these indicators, regarded as functions of effective model parameters (4). It is possible to appreciate that the combined use of critical indicators $\tilde{S}_{m i x}$ and $\tilde{S}_{l o c}$ (see the panels in the second and in the third row of Figure 3) allows one to distinguish the different phases clearly. It is worth noticing that, as the number of particles employed to perform the exact numerical diagonalization of Hamiltonian (1) is limited $\left(N_{a}=N_{b}=15\right)$, some finite size effects are present, which affect the "ideal" phase diagram illustrated in Figure 2. More prominently, the border between phase $\mathrm{M}$ and phase PL (line $\alpha=-1$ in the thermodynamic limit) has given way to a hyperbole-like border, which allows phase $\mathrm{M}$ to invade the half-plane $\alpha<-1$ (of course, for sufficiently small values of $\beta$ ).

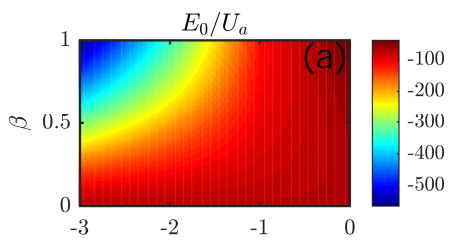

$\tilde{S}_{\operatorname{mix}}^{\alpha}$

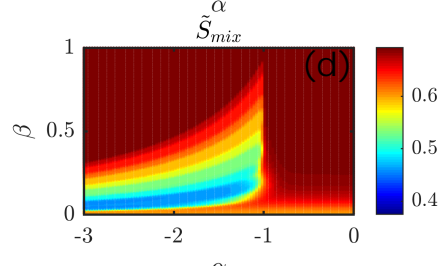

$\tilde{S}_{l o c}^{\alpha}$

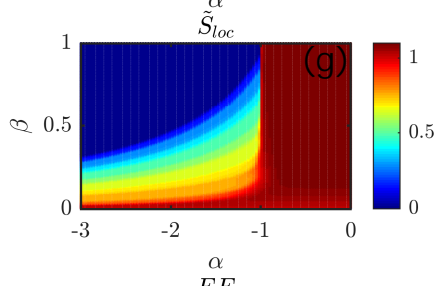

$E E$

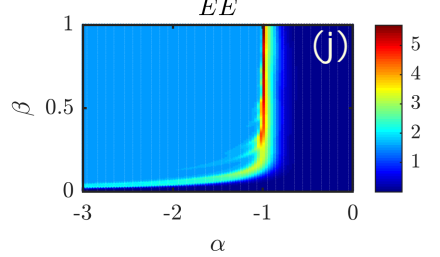

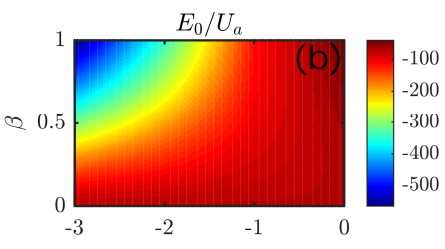

$\tilde{S}^{\alpha}$

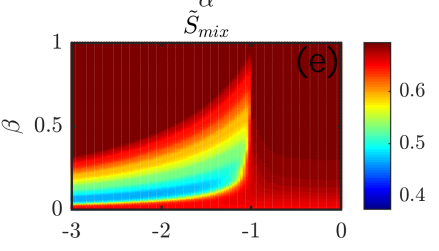

$\tilde{S}_{l o c}^{\alpha}$

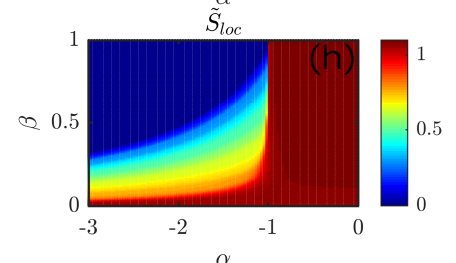

$E E$

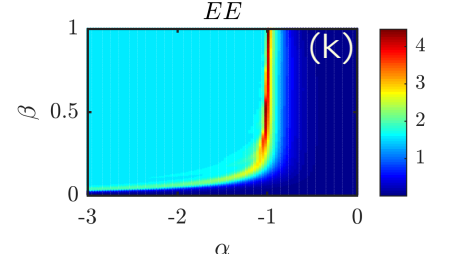

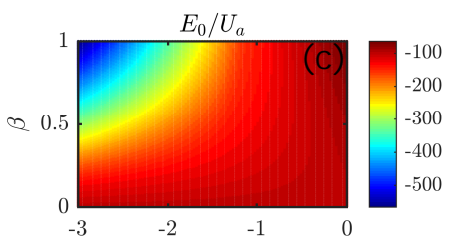

$\tilde{S}^{\alpha}$

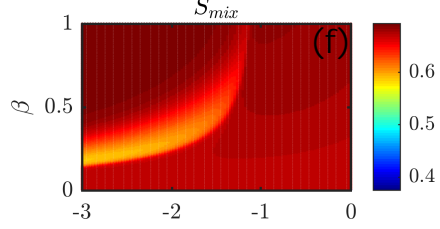

$\tilde{S^{\prime}}$

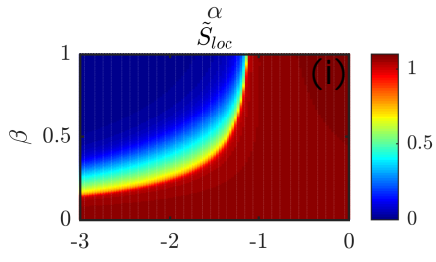

$\stackrel{\alpha}{E E}$

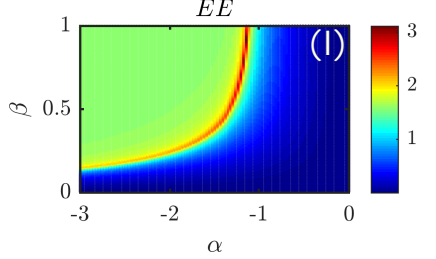

Figure 3. Each row illustrates the behavior of a genuinely quantum indicator as a function of model parameters $\alpha$ and $\beta$. Going from left to right, plots correspond to $T / U_{a}=0,0.02$, and 0.50 , where $T:=T_{a}=T_{b}$. (a-c): ground-state energy $E_{0} / U_{a}(11)$. (d-f): quantum version of the entropy of mixing, $\tilde{S}_{\text {mix }}$ (8). (g-i): quantum version of the entropy of location $\tilde{S}_{l o c}(9)$. (j-l): entanglement between the two condensed species, $E E$ (13). Model parameters $N_{a}=N_{b}=15, U_{a}=1, U_{b} \in[0,1] \Rightarrow \beta \in[0,1]$, and $\alpha \in[-3,0]$ were used. Each plot includes more than 20k points [41], corresponding to as many numerical diagonalizations of Hamiltonian (1).

The last row of Figure 3 illustrates the behavior of EE. The transition M-PL can be easily recognized, while the border PL-SM cannot be appreciated (as already noticed in [21]).

Eventually, as is visible in the first row of Figure 3, the ground-state energy $E_{0}$ as such does not allow for a direct identification of the various phases because its non-analytic character is better 
highlighted by its first- and second-order derivatives. This aspect will be discussed in Section 4 and illustrated in Figure 4, where the derivatives of $E_{0}$ with respect to $\alpha$ and $\beta$ (regarded, in turn, as functions thereof) are used to reconstruct the phase diagram effectively. Each column corresponds to a certain value of the tunneling amplitudes $T_{a}$ and $T_{b}$. Going from left to right, the latter increase, a circumstance that favors boson delocalization and determines the blurring of the phase diagram illustrated in Figure 2 (see [21] for further details).
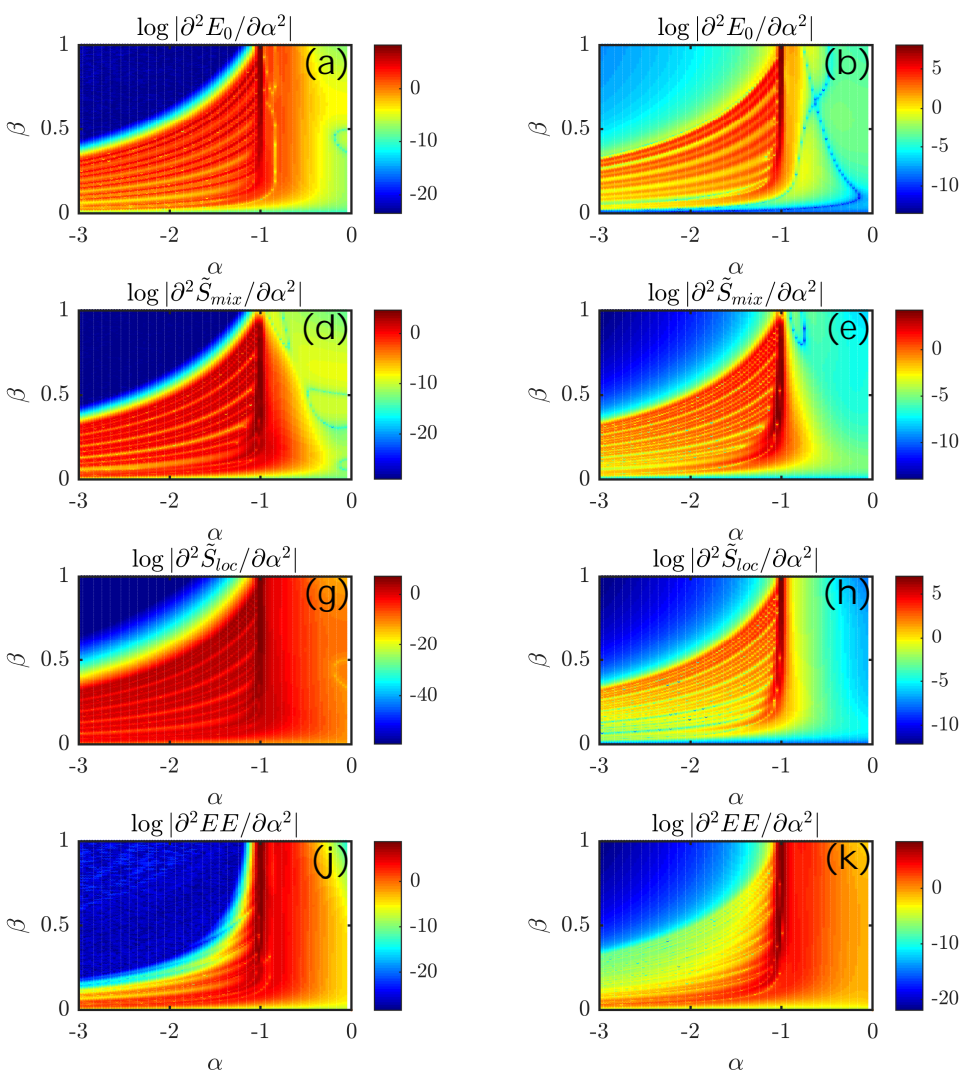
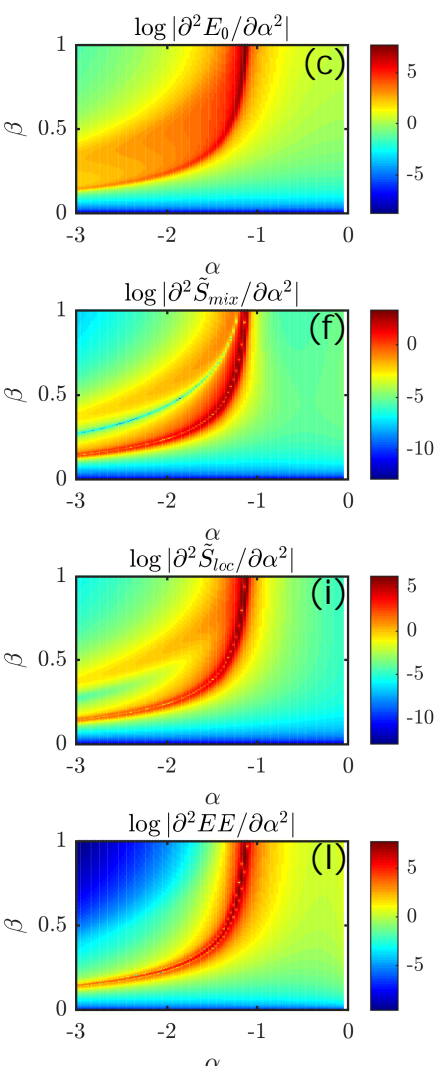

Figure 4. Each row illustrates the behavior of a genuinely quantum indicator as a function of model parameters $\alpha$ and $\beta$. Going from left to right, plots correspond to $T / U_{a}=0,0.02$, and 0.50 , where $T:=T_{a}=T_{b} .(\mathbf{a}-\mathbf{c})$ : second derivative of the ground-state energy $E_{0} .(\mathbf{d}-\mathbf{f})$ : second derivative of the quantum version of the entropy of mixing, $\tilde{S}_{m i x} .(\mathbf{g}-\mathbf{i})$ : second derivative of the quantum version of the entropy of location $\tilde{S}_{l o c}$. $(\mathbf{j}-\mathbf{l})$ : second derivative of the entanglement between the two condensed species, EE. Model parameters $N_{a}=N_{b}=15, U_{a}=1, U_{b} \in[0,1] \Rightarrow \beta \in[0,1]$, and $\alpha \in[-3,0]$ were used. Each plot includes more than 20k points [41], corresponding to as many numerical diagonalizations of Hamiltonian (1).

\section{Beyond the Continuous-Variable Picture: Emergence of the Quantum-Granularity}

The analytic treatment reviewed in Section 3 and based on the CV picture allows one to find all the phases that are possibly exhibited by the two species mixture in a rather straightforward way. The resulting phase diagram (see Figure 2) and the associated characteristic quantities (see Table 1) provide a full overview of the different ways in which the two quantum fluids can rearrange among available sites and allows one to recognize critical lines in the $(\alpha, \beta)$ plane.

Nevertheless, this semiclassical approximation scheme cannot accurately describe the ultraquantum effects exhibited by the system when boson populations $N_{a}$ and $N_{b}$ are finite and tunneling processes very weak. In these cases, in fact, a small variation of control parameters $\alpha$ and $\beta$ (see the Formula (4)) may not result in a smooth variation of the system's ground state.

To better clarify this circumstance, we begin with considering the central and the right panels of Figure 1. In the thermodynamic limit, one loses track of the quantum-granularity characterizing 
bosonic particles, and phase PL can be thought of as a collection of states that, upon increasing $|\alpha|$, smoothly approach the supermixed-soliton configuration. Therefore, in this scenario, the majority species gradually localizes upon increasing the interspecies attraction, meaning that the outer green bars in the central panel of Figure 1 are smoothly reabsorbed by the emerging supermixed soliton.

In this section, both by means of exact numerical computations and by developing a suitable analytic framework, we show that this smooth and elementary picture is no longer valid for finite values of boson populations $\left(N_{a}\right.$ and $N_{b}$ ) and for sufficiently low values of $T_{a}$ and $T_{b}$. In these regions of the parameter space, the discrete character of the interwell boson-exchange mechanism emerges, and the system discloses some new effects ensuing from the granularity of its constituents. Figure 5 provides a pictorial representation of this phenomenology.
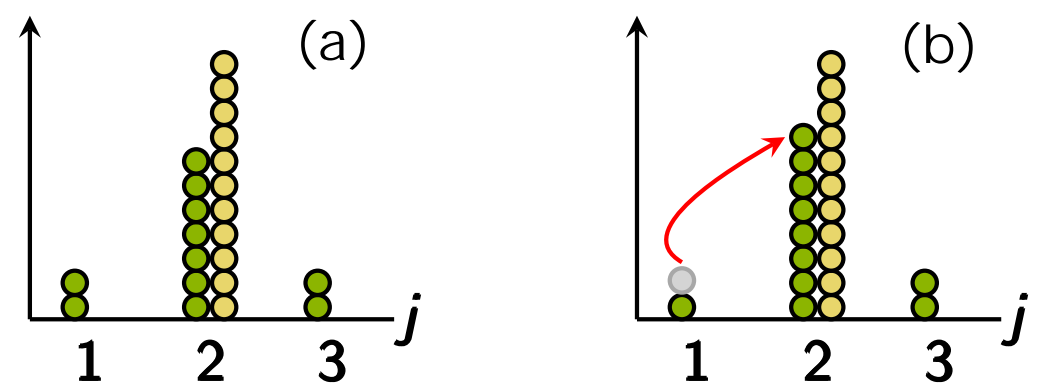

Figure 5. Pictorial representation of the discrete character of the interwell boson exchange. (a): macroscopic configuration of the system for a certain choice of model parameters. A small variation of control parameters $\alpha$ and $\beta$ may [panel (b)] or may not modify it. The fact that the supermixed soliton can gain or loose a boson at a time upon varying a control parameter is what we mean with the term "quantum-granularity". Green and yellow circles represent species- $a$ and species- $b$ bosons respectively. The gray circle represent a species- $a$ boson which is being transferred to the supermixed soliton.

\subsection{Exact Numerical Results}

The emergence of the aforementioned "quantum-granularity" in the phenomenology of the discussed system can be appreciated by resorting to the quantum indicators already introduced in Section 3.2 and including the ground-state energy, the entropy of mixing, the entropy of location, and the entanglement entropy. In Figure 4, we illustrate their second derivatives with respect to control parameter $\alpha$, where, for the sake of simplicity, we set $T:=T_{a}=T_{b}$. It is clear that, in the region of the $(\alpha, \beta)$ plane corresponding to phase PL, a staircase-like structure is present for sufficiently low values of $T$ (see the left and central columns of Figure 4). Conversely, this peculiar property is absent when tunneling is large enough (see the right column of Figure 4), a circumstance that can be explained in terms of the delocalizing effect of hopping processes, which tend to smooth down transitions and sharp features of the phase diagram [17-19,21].

The presence of this staircase-like structure in the central region of the $(\alpha, \beta)$ plane is due to the fact that, the hopping amplitude being small, the system responds to small variations of control parameters in a highly non-linear way. As will be explained in Section 4.2 by means of a simple analytic treatment, when tunneling terms tend to zero, phase PL (which, in the CV picture, can be thought of as a collection of states which transform in a smooth way when $\alpha$ and $\beta$ are varied) gives way to a sequence of stripes in the $(\alpha, \beta)$ plane within which the ground-state configuration proves to be rather rigid upon small variations of $\alpha$ and $\beta$ themselves. The transition between any two such stripes represents an abrupt change in the ground-state configuration and corresponds to the kind of boson rearrangement pictorially illustrated in Figure 5.

The staircase-like structure corresponding to jerky transfers of bosons from/to the site hosting the supermixed soliton is evident also in terms of the energy fingerprint of the system. The latter, 
i.e., the set of the first excited energy levels, are shown in Figure 6 for different values of the hopping amplitudes.
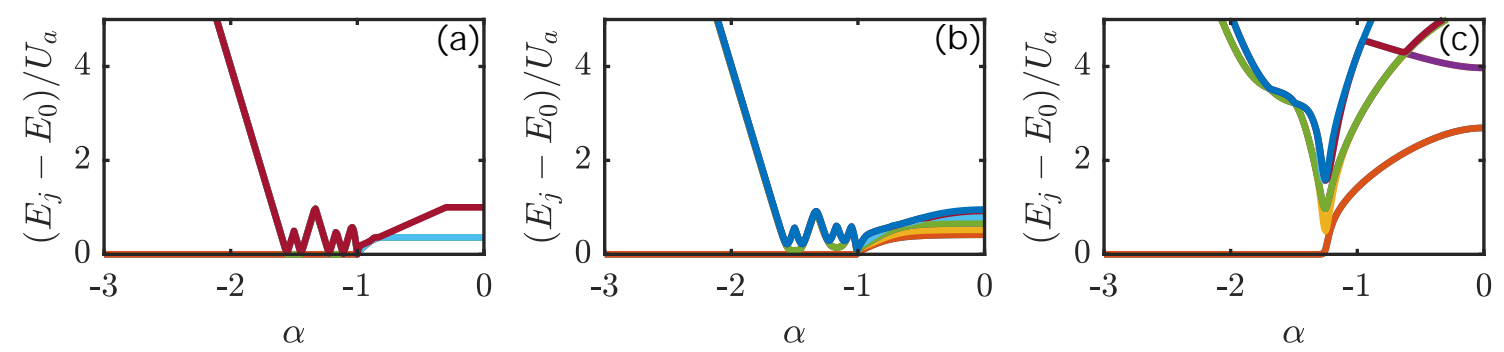

Figure 6. First eight excited energy levels, obtained by means of an exact numerical diagonalization of Hamiltonian (1), for $T:=T_{a}=T_{b}=0,0.02,0.50$ in panel (a-c), respectively. Model parameters $N_{a}=N_{b}=15, U_{a}=1, U_{b}=0.36 \Rightarrow \beta=0.6$, and $W \in[-1.8,0] \Rightarrow \alpha \in[-3,0]$ were chosen. Each color corresponds to a different energy level.

In particular, if the hopping amplitudes are sufficiently small (see the left and central panels of Figure 6), the energy-level structure in the region of the $(\alpha, \beta)$ plane between phase $M$ and phase $S M$ features sharp peaks. With reference to the aforementioned figure, where $\beta$ was set to 0.6 , the staircase-like structure is present for $-1.6 \leq \alpha \leq-1$. The number of peaks in the energy spectrum corresponds to that of the stripes that one crosses while walking along a straight line at $\beta=$ const in the $(\alpha, \beta)$ plane. Similarly, the number of valleys visible in the energy spectrum corresponds to that of stripes borders crossed by the constant $\beta$ pathway. The sequence of stripes whose borders correspond to jerky boson transfers (of the type sketched in Figure 5) can be clearly appreciated also in Figure 7 , which has been derived within a fully-analytic framework (see Section 4.2 for details).

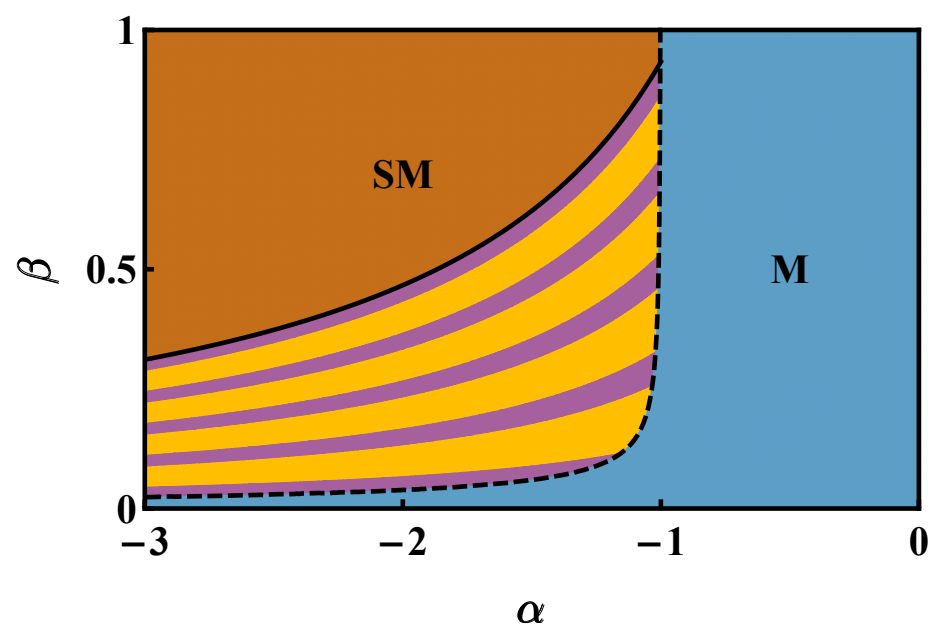

Figure 7. Map of the system's minimum energy configurations. It corresponds to the graphical representation of the set of inequalities derived in Section 4.2. More specifically: the solid black (dashed) line corresponds to the condition (23) and (28), while the set of purple (yellow) stripes is given by the condition (24) and (25). Model parameters $N_{a}=N_{b}=15, U_{a}=1, U_{b} \in[0,1] \Rightarrow \beta \in[0,1]$, $\alpha \in[-3,0]$, and $T_{a}=T_{b}=0$ were used.

If hopping amplitudes $T_{a}$ and $T_{b}$ exceed a certain threshold, the discrete character of the interwell boson exchange fades away, and the energy levels $E_{j}(\alpha)$, regarded as functions of control parameter $\alpha$, become well behaved (see the right panel of Figure 6).

Another effective indicator that can provide insight into the jerky transfers of bosons from/to the supermixed soliton is represented by $\mathcal{D}\left(E_{0}\right)$, the degeneracy of the ground-state level when $T_{a}=T_{b}=0$. We recall, in this regard, that, as soon as the tunneling is non-vanishing, the ground 
state of Hamiltonian (1) becomes unique and is not degenerate [42], although it can take the form of a superposition of a few macroscopically different configurations (a Schrödinger-cat state) [18,43]. As we shall discuss, such a superposition of different Fock states, although being non-degenerate, bears the memory of the value of $\mathcal{D}\left(E_{0}\right)$ that one would have if hopping processes were suppressed, since $\mathcal{D}\left(E_{0}\right)$ at $T_{a}=T_{b}=0$ corresponds to the number of macroscopic configurations that constitute the non-degenerate Schrödinger-cat state at small, but finite tunnelings.

The value of $\mathcal{D}\left(E_{0}\right)$, computed along a path in the $(\alpha, \beta)$ plane featuring $\beta=$ const, is illustrated in Figure 8. At the chosen value of $\beta$, for $\alpha<2.3$, the system's ground state takes the form of a supermixed soliton whose degeneracy $\mathcal{D}$ is three, because three is the number of its possible positions in the trimer. For $-1<\alpha<0$, the configuration that minimizes the (expectation value of) Hamiltonian (1) is the uniform and mixed one. The latter is such that there are $N_{a} / 3$ species- $a$ and $N_{b} / 3$ species- $b$ bosons in each site. If, as in the case of Figure $8, N_{a}$ and $N_{b}$ are integer multiples of the number of lattice sites, there exists just one state that minimizes energy (1), and accordingly, the associated degeneracy $\mathcal{D}\left(E_{0}\right)$ is unitary.

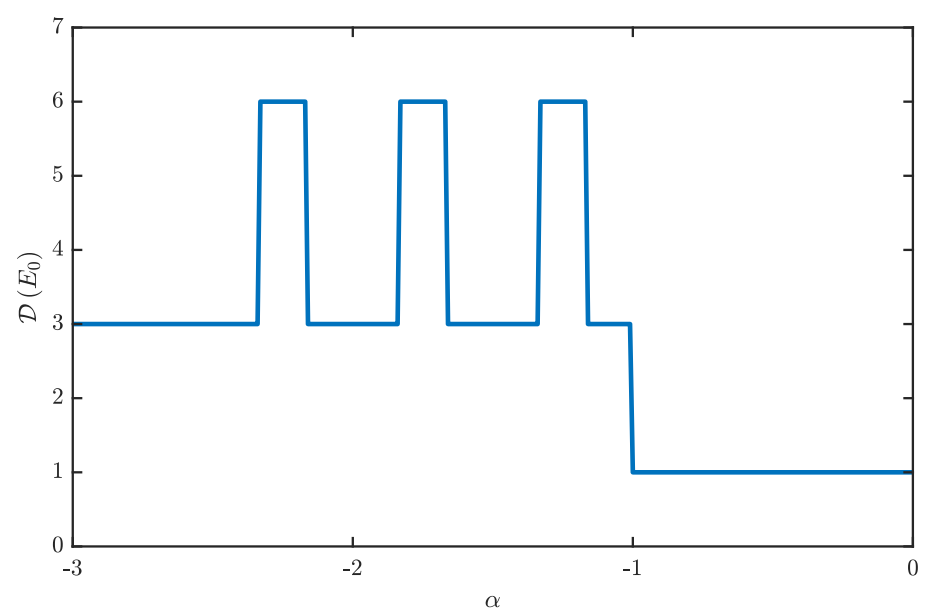

Figure 8. Degeneracy of the ground-state level $E_{0}$, obtained by means of an exact numerical diagonalization of Hamiltonian (1), for $T:=T_{a}=T_{b}=0$. Model parameters $N_{a}=N_{b}=15$, $U_{a}=1, U_{b}=0.16 \Rightarrow \beta=0.4$, and $W \in[-1.2,0] \Rightarrow \alpha \in[-3,0]$ were chosen. Each jump discontinuity corresponds to a change in the ground state structure of the type illustrated in Figure 5.

For $-2.3<\alpha<-1$, the system ground state transforms from the mixed to the supermixed one in such a way that bosons are transferred to the emerging supermixed soliton in the jerky fashion sketched in Figure 5. Accordingly, the degeneracy of the ground-state level alternatively takes the values three and six, depending on the number of species- $a$ bosons that are not part of the supermixed soliton. To better clarify this property, we observe that, in the region of the phase diagram corresponding to phase PL, at $T_{a}=T_{b}=0$, the ground state of Hamiltonian (1) is made up of Fock states of the type:

$$
\left|N_{1}, N_{2}, N_{3}, M_{1}, M_{2}, M_{3}\right\rangle=\left|N_{a}-N_{2}-N_{3}, N_{2}, N_{3}, N_{b}, 0,0\right\rangle
$$

where $N_{2}<N_{a}-N_{2}-N_{3}$ and $N_{3}<N_{a}-N_{2}-N_{3}$. In light of this, one can immediately conclude that the degeneracy of the associated energy level, i.e., the number of possible permutations of the quantum numbers that come into play, is three when $N_{2}=N_{3}$, and it is six when $N_{2} \neq N_{3}$. With reference to Figure 7, which was obtained by means of the fully-analytic framework discussed in Section 4.2, purple (yellow) stripes are associated with $\mathcal{D}=6(\mathcal{D}=3)$, while, as already explained, in the regions $\mathrm{SM}$ and $\mathrm{M}, \mathcal{D}$ takes the values three and one, respectively. The fact that purple and yellow stripes have different widths will be explained by the simple analytic framework presented in Section 4.2.

The discussed mechanism of jerky interwell boson transfer is present not only at $T=0$, but it persists also for finite values of tunnelings. To better illustrate this circumstance, we refer to Figure 9 , 
where we plot the second derivative of the ground-state energy (11) with respect to control parameter $\alpha$ (left panel) and the entropy:

$$
S=-\sum_{\vec{N}} \sum_{\vec{M}}|c(\vec{N}, \vec{M})|^{2} \log |c(\vec{N}, \vec{M})|^{2}
$$

of the probability distribution associated with coefficients (10) (right panel). Both plots, which are referred to the $\left(\alpha, T / U_{a}\right)$ plane, clearly show the presence of lobes for small values of $T / U_{a}$ and for $-2.4<\alpha<-1$. More specifically, as regards the left plot, one can appreciate a sequence of six lobes (depicted in green), which correspond to a sequence of Fock states of the type:

$$
\begin{aligned}
\left|\psi_{0}\right\rangle_{6} \approx & \frac{1}{\sqrt{6}}\left[\left|N_{a}-N_{2}-N_{3}, N_{2}, N_{3}, N_{b}, 0,0\right\rangle+\left|N_{a}-N_{2}-N_{3}, N_{3}, N_{2}, N_{b}, 0,0\right\rangle+\right. \\
& +\left|N_{2}, N_{a}-N_{2}-N_{3}, N_{3}, 0, N_{b}, 0\right\rangle+\left|N_{3}, N_{a}-N_{2}-N_{3}, N_{2}, 0, N_{b}, 0\right\rangle+ \\
& \left.+\left|N_{2}, N_{3}, N_{a}-N_{2}-N_{3}, 0,0, N_{b}\right\rangle+\left|N_{3}, N_{2}, N_{a}-N_{2}-N_{3}, 0,0, N_{b}\right\rangle\right]
\end{aligned}
$$

for $N_{2} \neq N_{3}$, and of the type:

$$
\begin{gathered}
\left|\psi_{0}\right\rangle_{3} \approx \frac{1}{\sqrt{3}}\left[\left|N_{a}-N_{2}-N_{3}, N_{2}, N_{3}, N_{b}, 0,0\right\rangle+\left|N_{2}, N_{a}-N_{2}-N_{3}, N_{3}, 0 N_{b}, 0\right\rangle+\right. \\
\left.+\left|N_{2}, N_{3}, N_{a}-N_{2}-N_{3}, 0,0, N_{b}\right\rangle\right]+
\end{gathered}
$$

for $N_{2}=N_{3}$, where the symbol " $\approx$ " was used to recall that, when $T>0$, many other Fock states $|\vec{N}, \vec{M}\rangle$ enter into the expression of $\left|\psi_{0}\right\rangle$, but their weights $|c(\vec{N}, \vec{M})|^{2}$ (see (10)) in the linear combination $\left|\psi_{0}\right\rangle=\sum_{\vec{N}} \sum_{\vec{M}} c(\vec{N}, \vec{M})|\vec{N}, \vec{M}\rangle$ are very small if the ratio $T / U_{a}$ is, in turn, small. Going from left to right in both plots of Figure 9, for small enough values of $T / U_{a}$, the quantum number $N_{a}-N_{2}-N_{3}$, which correspond to the number of species- $a$ bosons in the supermixed soliton, takes the value of 15 for $\alpha<-2.4$ (SM configuration) and the value of five for $\alpha>-1$. More interestingly, for $-2.4<\alpha<-1$, it takes the sequence of values 14,13,12,11,10,9. Accordingly, the system ground state alternately takes the form of the state (16) and state (17). This sequence of six different ground states corresponds to that of the six green lobe-like domains in the bottom part of the left panel of Figure 9 and to that of the blue lobe-like domains in the bottom part of the right panel of Figure 9. Notice, in this regard, that the domains corresponding to the cases $N_{2}=N_{3}$ are bigger, i.e., they are wider and persist for bigger values of $T / U_{a}$. Conversely, the lobes corresponding to the cases $N_{2} \neq N_{3}$ are narrower and are more easily disrupted by tunneling. The different widths of the lobes for $N_{2}=N_{3}$ and of those for $N_{2} \neq N_{3}$ will be explained in Section 4.2 (by means of a simple analytical model), while their different heights can be explained by means of an analogy with the superfluid-Mott insulator transition. Note that, also, these two kinds of lobes visible in the right panel of Figure 9 alternately take the values of $S \approx \log 6$ and $S \approx \log 3$, in that the number of macroscopic components present in the non-degenerate Schrödinger-cat-like states of the type (16) and (17) bears memory of the degeneracy $\mathcal{D}\left(E_{0}\right)$ of the ground state if the tunneling $T$ was suppressed.

In order to highlight the analogy with the superfluid-Mott insulator transition, we start by looking at the trimer system as if it were made up of two parts. One corresponds to the site where the supermixed soliton is emerging: it includes $N_{a}-N_{2}-N_{3}$ species- $a$ bosons and $N_{b}$ species- $b$ bosons. The other part corresponds to the remaining two sites, hosting, in total, $N_{2}+N_{3}$ species- $a$ bosons and zero species- $b$ bosons. As $N_{a}-N_{2}-N_{3}$ can be much bigger than $N_{2}+N_{3}$, the macroscopically occupied site can be thought of as a reservoir of species- $a$ bosons, and the remaining two sites can be regarded as a two-well system including just one bosonic species (instead of a binary mixture), which is in contact with a particle reservoir. In this perspective, the interspecies attraction $W$ and hence 
effective control parameter $\alpha$ (see Formula (4)), plays the role of an effective chemical potential, as it can control the release/absorption of species- $a$ bosons from/to the particle reservoir.
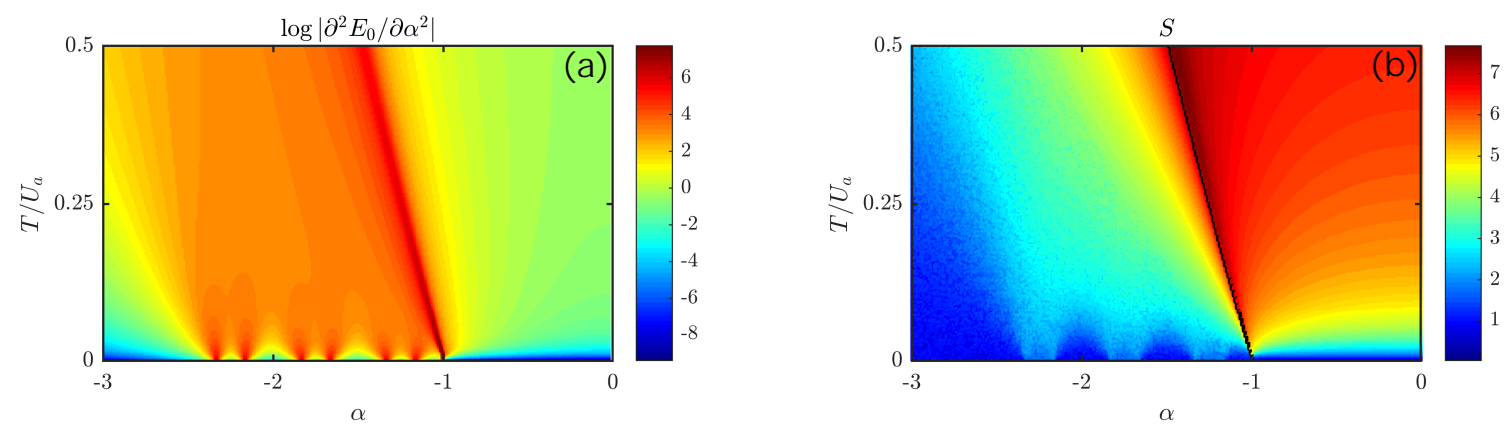

Figure 9. The mechanism of jerky interwell boson transfer is present provided that tunneling $T$ is small enough. (a): second derivative of the ground-state energy (11) with respect to control parameter $\alpha$. (b): entropy (15) of the probability distribution associated with coefficients $|c(\vec{N}, \vec{M})|^{2}$ (see Formula (10)). The black line corresponds to the border of the stability region (20) of the mixed configuration. Notice that, unlike Figures 3 and 4 , these plots are referred to the $\left(\alpha, T / U_{a}\right)$ plane, instead of the $(\alpha, \beta)$ plane. Model parameters $N_{a}=N_{b}=15, U_{a}=1, U_{b}=0.16 \Rightarrow \beta=0.4, T_{a}=T_{b}=: T \in[0,0.5]$, and $\alpha \in[-3,0]$ were used. Each plot includes more than $75 \mathrm{k}$ points [41], corresponding to as many numerical diagonalizations of Hamiltonian (1).

Notice that there is a profound difference between states (17) and states (16). Concerning the effective two-well potential resulting from the exclusion of the macroscopically occupied site (which plays the role of particle reservoir), the former are marked by a commensurate filling, while the second feature an incommensurate filling. As a consequence, lobes corresponding to the case of $N_{2}=N_{3}$ play the role of Mott lobes, while those corresponding to the case $N_{2} \neq N_{3}$ correspond to superfluid lobes, as one species- $a$ boson is shared between the sites of the effective two-well potential.

Interestingly, both states (17) and states (16) seem to undergo a deep change when $T$ exceeds a certain threshold, which is different in the two cases $\left(T / U_{a} \approx 0.02\right.$ and $\approx 0.01$, respectively). In the first case, as $N_{2}=N_{3}$ (commensurate filling), the analogy with the superfluid-Mott insulator transition suggests that, increasing the ratio $T / U_{a}$, bosons tend to be delocalized, and the system switches from the Mott to the superfluid phase. Concerning the other family of states, (16), featuring $N_{2} \neq N_{3}$, the interpretation is more delicate. This is because they are already endowed with a superfluid character, as one boson is shared by the two sites of the resulting effective system. Although this property deserves further investigation (we expect that an increasingly rich structure of "superfluid lobes" necessarily emerges when the number of lattice sites increases), it is possible to conjecture that, crossing the border of such a lobe, the system switches from a weaker to a stronger type of superfluidity. In fact, the states (16) are forcefully superfluid, even for $T \rightarrow 0^{+}$, because of the extra boson expelled by the supermixed soliton and injected into the effective two-well potential. Nevertheless, the superfluid character of state (16) is strongly dammed by the fact that it includes just six Fock states (actually two, if one neglects the possible ways to permute the position of the particle reservoir), and therefore, it is far from being of the type:

$$
\left|\psi_{0}\right\rangle \propto\left(A_{2}^{\dagger}+A_{3}^{\dagger}\right)^{N_{2}+N_{3}}|0,0\rangle,
$$

the latter representing the exact ground state of a two-well $\mathrm{BH}$ Hamiltonian featuring $U / T \rightarrow 0$ and hosting $\mathrm{N}_{2}+N_{3}$ species- $a$ bosons. This circumstance would reasonably explain the presence of small lobes in both panels of Figure 9. In the same spirit of [44], where suitable squeezing indicators were introduced to detect lobe-like structures in an asymmetric BH-dimer Hamiltonian, we introduce indicator: 


$$
\Delta n=\frac{1}{2}\left(2 N_{\max }-N_{i}-N_{j}\right),
$$

where $N_{\max }:=\max _{k \in\{1,2,3\}}\left\{N_{k}\right\}$ and $N_{i}, N_{j} \in\left\{N_{1}, N_{2}, N_{3}\right\}-\left\{N_{\max }\right\}$, which corresponds to the average species- $a$ bosons imbalance between the site hosting the supermixed soliton and the sites of the remaining two-well system. As is visible in Figure 10, where the expectation value $\langle\Delta n\rangle=\left\langle\psi_{0}|\Delta n| \psi_{0}\right\rangle$ is plotted, when $T / U_{a}$ is small enough, a sequence of lobe-like domains is present, which corresponds to the sequence of values 15 (SM configuration), 13.5 (first superfluid-like lobe), 12 (first Mott-like lobe), 10.5, and so on.

We conclude this section by recalling that it is possible to find, either within the CV picture [21] or by means of the dynamical-algebra method [31], the region of the parameters space where the mixed configuration (the one sketched in the leftmost panel of Figure 1) is stable. It is given by inequality:

$$
\alpha>-\sqrt{\left(1+\frac{9}{2} \frac{T_{a}}{U_{a} N_{a}}\right)\left(1+\frac{9}{2} \frac{T_{b}}{U_{b} N_{b}}\right)}
$$

whose border, in the $\left(\alpha, T / U_{a}\right)$ plane, corresponds to the black line in the right panel of Figure 9 . Interestingly, one can notice that, while approaching this border from the right, the entropy (15) associated with the ground state significantly increases and takes the maximum value exactly at the value of $\alpha$ where the mixed configuration gives way to a configuration of the type (14).

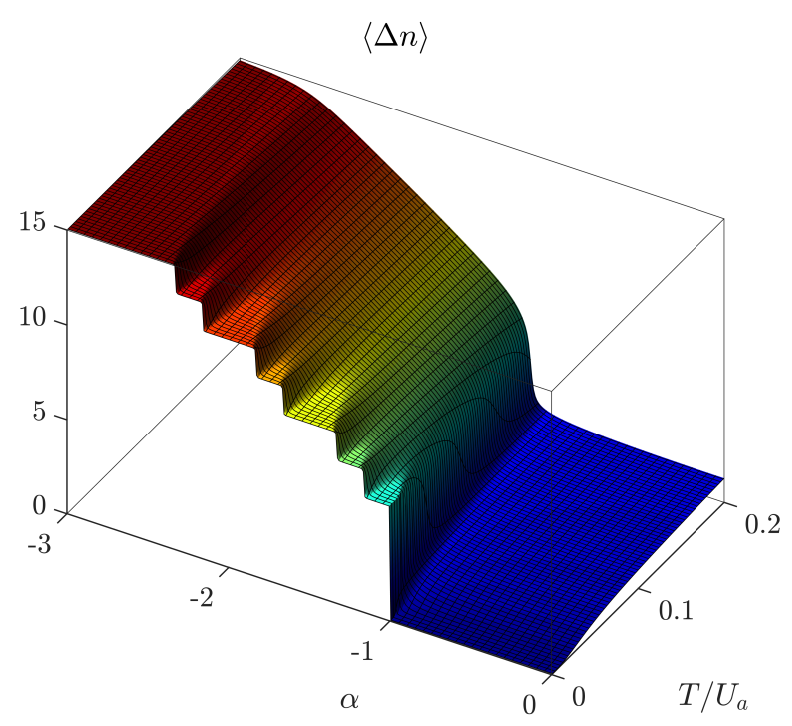

Figure 10. Expectation value $\langle\Delta n\rangle=\left\langle\psi_{0}|\Delta n| \psi_{0}\right\rangle$ of operator imbalance operator $\Delta n$ (see Formula (19)) as a function of $\alpha$ and $T / U_{a}$. The mechanism of jerky interwell boson transfer is present provided that tunneling $T$ is small enough (compare the staircase-like structure for $T / U_{a} \rightarrow 0$ with the slide-like appearance for $T / U_{a} \approx 0.2$ ). Notice that, unlike Figures 3 and 4 , these plots are referred to the $\left(\alpha, T / U_{a}\right)$ plane, instead of the $(\alpha, \beta)$ plane. Notice also that, unlike Figure 9 , the range of $T / U_{a}$ is $[0,0.2]$ in order to better appreciate the presence of lobe-like regions. Model parameters $N_{a}=N_{b}=15$, $U_{a}=1, U_{b}=0.16 \Rightarrow \beta=0.4, T_{a}=T_{b}=: T \in[0,0.5]$, and $\alpha \in[-3,0]$ were used. The plot includes more than $60 \mathrm{k}$ points [41], corresponding to as many numerical diagonalizations of Hamiltonian (1). Color is used to better emphasize the features of the surface: blue (red) corresponds to $\langle\Delta n\rangle=0$ $(\langle\Delta n\rangle=15)$.

\subsection{Analytic Treatment}

We present a simple, but effective analytical treatment, capable of capturing the presence of the staircase-like structure in the central region of the $(\alpha, \beta)$ plane (see Figure 4$)$. By means of fully-analytic computations, we derive, for $T=0$, a set of inequalities giving the stability region not only of the 
mixed and of the supermixed configurations, but also of each intermediate configuration of the type (14). The graphical representation of these inequalities is shown in Figure 7, which effectively mimics the scenario illustrated in Figure 4, obtained, in turn, by sweeping model parameters and numerically diagonalizing Hamiltonian (1).

Let us consider a supermixed-soliton configuration. The associated energy, for $T=0$, reads:

$$
E(S M)=\frac{U_{a}}{2} N_{a}\left(N_{a}-1\right)+\frac{U_{b}}{2} N_{b}\left(N_{b}-1\right)+W N_{a} N_{b} .
$$

The first state belonging to the staircase-like structure differs from a supermixed-soliton state because one species- $a$ boson has left the macroscopically occupied site and has moved to the remaining two-well system. The energy of this configuration reads:

$$
E\left(S M-1_{a}\right)=\frac{U_{a}}{2}\left(N_{a}-1\right)\left(N_{a}-2\right)+\frac{U_{b}}{2} N_{b}\left(N_{b}-1\right)+W\left(N_{a}-1\right) N_{b} .
$$

By solving the inequality $E(S M)<E\left(S M-1_{a}\right)$, one obtains that the supermixed configuration ceases to be the energetically favorable one for:

$$
\alpha>\frac{1}{\beta}\left(\frac{1}{N_{a}}-1\right) .
$$

This condition corresponds to the solid black line in Figure 7 and allows one to recognize the border between the region of SM states and the first element of the staircase-like structure. It is worth mentioning the fact that it would be energetically unfavorable to remove a species- $b$ (instead of a species- $a)$ boson from the supermixed soliton. The condition $E\left(S M-1_{a}\right)<E\left(S M-1_{b}\right)$ is indeed always verified in the chosen range $\beta \in[0,1]$ because of the asymmetric role of species- $a$ and species- $b$ parameters in the definition of $\beta$ (see Formula (4)). State $\left|N_{a}-1,1,0, N_{b}, 0,0\right\rangle$ is the actual system ground state provided that the condition (23) is satisfied and that $E\left(S M-1_{a}\right)<E\left(S M-2_{a}\right)$. The latter inequality corresponds to the border between the upper purple stripe and its neighboring yellow stripe in Figure 7.

One can easily generalize this reasoning in order to find the condition under which a state of the type (14) and such that $K_{a}=N_{2}+N_{3}$ species- $a$ bosons have left the supermixed soliton is the actual system's ground state. One needs to distinguish two cases: $K_{a}$ odd and $K_{a}$ even. After some straightforward algebra, it turns out that the aforementioned state, whose energy is $E\left(S M-K_{a}\right)$, is the actual ground state provided that:

$$
\begin{gathered}
\frac{1}{\beta}\left(\frac{3\left(K_{a}-1\right) / 2+1}{N_{a}}-1\right)<\alpha<\frac{1}{\beta}\left(\frac{3\left(K_{a}-1\right) / 2+2}{N_{a}}-1\right) \quad \text { if } K_{a} \text { is odd, } \\
\frac{1}{\beta}\left(\frac{3 K_{a} / 2-1}{N_{a}}-1\right)<\alpha<\frac{1}{\beta}\left(\frac{3 K_{a} / 2+1}{N_{a}}-1\right) \quad \text { if } K_{a} \text { is even. }
\end{gathered}
$$

With reference to Figure 7, the former (latter) set of inequalities corresponds to the set of purple (yellow) stripes. Notice also that these simple analytical expressions perfectly capture the fact that yellow stripes are two times wider than purple stripes or, in other words, that, in Figure 8, the pulses with degeneracy $\mathcal{D}\left(E_{0}\right)=6$ are two times narrower than those with degeneracy $\mathcal{D}\left(E_{0}\right)=3$. The same reasoning, of course, accounts for the different widths of the superfluid-like and Mott-insulator-like lobes of Figure 9 (see the relevant discussion in Section 4.1).

It is known from the theory developed in [21] and reviewed in Section 3 that, when $\alpha$ approaches the value $\approx-1$, the system ground state sharply switches to the uniform and mixed $(M)$ configuration, featuring $N_{a} / 3$ species- $a$ and $N_{b} / 3$ species- $b$ bosons in each well. This dramatic change in the structure of the ground state corresponds, in the thermodynamic limit, to the transition M-PL (see Section 3). To derive the condition under which the mixed configuration, featuring energy: 


$$
E(M)=3 \frac{U_{a}}{2} \frac{N_{a}}{3}\left(\frac{N_{a}}{3}-1\right)+3 \frac{U_{b}}{2} \frac{N_{b}}{3}\left(\frac{N_{b}}{3}-1\right)+3 W \frac{N_{a}}{3} \frac{N_{b}}{3},
$$

becomes energetically favorable, one needs to solve the inequality $E(M)<E\left(S M-K_{a}\right)$, giving:

$$
\alpha>\left(\frac{3 K_{a}}{4 N_{a}}-\frac{1}{2}\right) \frac{1}{\beta}-\frac{N_{a} \beta}{2 N_{a}-3 K_{a}},
$$

and then impose that the critical value of $\alpha$ falls exactly where the lobe with energy $E\left(S M-K_{a}\right)$ would give way to the lobe with energy $E\left(S M-K_{a}-1\right)$. As a result, one obtains relation:

$$
\alpha^{*}=-\frac{\sqrt{\beta^{2} N_{a}^{2}+1}}{\beta N_{a}}
$$

giving the border between region $\mathrm{M}$ and the staircase-like structure (see black dashed line in Figure 7) and relation:

$$
K_{a, \max }=\frac{2}{3}\left(N_{a}-1-\sqrt{\beta^{2} N_{a}^{2}+1}\right)
$$

giving, for a certain value of $\beta$, the maximum number of species- $a$ bosons that can be subtracted from the supermixed soliton before abruptly switching to the uniform and mixed configuration (of course, as $K_{a, \max }$ must be an integer number, the use of the floor function is implicitly needed).

It is important to remark that the presence of the staircase-like structure that is observed for small values of $T$ and finite boson populations, $N_{a}$ and $N_{b}$, is not in contrast with the analysis developed within the CV picture (see [21] and its brief review in Section 3), but it is complementary to it. In fact, in the limit of large boson populations, one loses track of the quantum-granularity, which is responsible for the sequence of superfluid-like and Mott-insulator-like lobes, and one re-obtains the same expressions that were obtained by approximating boson populations with continuous variables. For example, one has that:

$$
\lim _{N_{a} \rightarrow+\infty} \alpha^{*}=-1,
$$

which corresponds to the M-PL border in the phase diagram illustrated in Figure 2, and also:

$$
\lim _{N_{a} \rightarrow+\infty} \frac{K_{a, \max }}{N_{a}}=\frac{2(1-\beta)}{3},
$$

which perfectly matches the results obtained within the CV picture (see Table 1 at the M-PL transition).

\section{Conclusions}

In this work, we investigated the quantum-granularity effect characterizing the formation of supermixed solitons in ring lattices. It occurred for small values of the tunneling parameters and consisted of a jerky transfer of bosons from/to the site hosting the supermixed soliton. Interestingly, we showed that it is possible to draw an analogy between the physics of a mixture trapped in a few well potential and that of the superfluid-Mott insulator transition. More specifically, we showed that, in certain regimes, the interspecies attraction played the role of an effective chemical potential and therefore controlled the release of bosons from a macroscopically occupied site, which, in turn, played the role of the particle reservoir.

In Section 2, we introduced the model, highlighting the fact that we considered a bosonic binary mixture featuring repulsive intraspecies and attractive interspecies couplings. In Section 3.1, we presented the system phase diagram, which was shown to be spanned by just two effective parameters, accounting for the ratio between inter- and intra-species couplings, and incorporating the possible asymmetry between bosonic species. Section 3.2 was devoted to the presentation of 
several quantum indicators, which were conveniently used to quantify the degree of localization and mixing of the two bosonic species, and the amount of quantum correlation (entanglement) between them. In Section 4, we pointed out that small hopping amplitudes were responsible for a discrete interwell boson exchange and hence for the emergence of a staircase-like structure in the central region of the phase diagram. For this purpose, in Section 4.1, we showed the behavior of different quantum indicators including, but not limited to the energy spectrum, various types of entropy, and the degree of degeneracy of the ground-state level. The interesting analogy with the mechanism of the superfluid-Mott insulator transition was also discussed. Eventually, in Section 4.2, we presented a simple, but effective analytic framework capable of capturing the emergence of the quantum-granularity effect and the ensuing properties. The rich sequence of Mott-like and superfluid-like lobes revealed for the ring trimer is expected to be present in larger sizes lattices. This aspect deserves further investigation, which we shall develop elsewhere.

Author Contributions: Conceptualization, A.R. and V.P.; Formal analysis, A.R.; Investigation, A.R. and V.P.; Software, A.R.; Supervision, V.P.; Writing—original draft, A.R.; Writing—review and editing, A.R. and V.P. All authors have read and agreed to the published version of the manuscript.

Funding: This research received no external funding.

Acknowledgments: Both authors warmly thank one of anonymous referees of [21] for prompting us to investigate the system in the low tunneling regime and in the presence of a finite number of bosons.

Conflicts of Interest: The authors declare no conflicts of interest.

\section{Abbreviations}

The following abbreviations are used in this manuscript:

BH Bose-Hubbard

CV Continuous-variable

SM Supermixed

PL Partially localized

M Mixed

EE Entanglement entropy

\section{References}

1. Modugno, G.; Modugno, M.; Riboli, F.; Roati, G.; Inguscio, M. Two Atomic Species Superfluid. Phys. Rev. Lett. 2002, 89, 190404. [CrossRef] [PubMed]

2. Chin, C.; Grimm, R.; Julienne, P.; Tiesinga, E. Feshbach resonances in ultracold gases. Rev. Mod. Phys. 2010, 82, 1225-1286. [CrossRef]

3. Myatt, C.J.; Burt, E.A.; Ghrist, R.W.; Cornell, E.A.; Wieman, C.E. Production of Two Overlapping Bose-Einstein Condensates by Sympathetic Cooling. Phys. Rev. Lett. 1997, 78, 586-589. [CrossRef]

4. Catani, J.; De Sarlo, L.; Barontini, G.; Minardi, F.; Inguscio, M. Degenerate Bose-Bose mixture in a three-dimensional optical lattice. Phys. Rev. A 2008, 77, 011603. [CrossRef]

5. Mishra, T.; Pai, R.V.; Das, B.P. Phase separation in a two-species Bose mixture. Phys. Rev. A 2007, 76, 013604. [CrossRef]

6. Jain, P.; Boninsegni, M. Quantum demixing in binary mixtures of dipolar bosons. Phys. Rev. A 2011, 83, 023602. [CrossRef]

7. Lingua, F.; Guglielmino, M.; Penna, V.; Capogrosso Sansone, B. Demixing effects in mixtures of two bosonic species. Phys. Rev. A 2015, 92, 053610. [CrossRef]

8. Suthar, K.; Angom, D. Optical-lattice-influenced geometry of quasi-two-dimensional binary condensates and quasiparticle spectra. Phys. Rev. A 2016, 93, 063608. [CrossRef]

9. Suthar, K.; Roy, A.; Angom, D. Fluctuation-driven topological transition of binary condensates in optical lattices. Phys. Rev. A 2015, 91, 043615. [CrossRef]

10. Buonsante, P.; Giampaolo, S.M.; Illuminati, F.; Penna, V.; Vezzani, A. Mixtures of Strongly Interacting Bosons in Optical Lattices. Phys. Rev. Lett. 2008, 100, 240402. [CrossRef] 
11. Roscilde, T.; Cirac, J.I. Quantum Emulsion: A Glassy Phase of Bosonic Mixtures in Optical Lattices. Phys. Rev. Lett. 2007, 98, 190402. [CrossRef] [PubMed]

12. Belemuk, A.; Chtchelkatchev, N.; Mikheyenkov, A.; Kugel, K. Quantum phase transitions and the degree of nonidentity in the system with two different species of vector bosons. New J. Phys. 2018, 20, 063039. [CrossRef]

13. Wang, W.; Penna, V.; Capogrosso-Sansone, B. Inter-species entanglement of Bose-Bose mixtures trapped in optical lattices. New J. Phys. 2016, 18, 063002. [CrossRef]

14. Mujal, P.; Juliá-Díaz, B.; Polls, A. Quantum properties of a binary bosonic mixture in a double well. Phys. Rev. A 2016, 93, 043619. [CrossRef]

15. Melé-Messeguer, M.; Julia-Diaz, B.; Guilleumas, M.; Polls, A.; Sanpera, A. Weakly linked binary mixtures of $F=1{ }^{87} \mathrm{Rb}$ Bose-Einstein condensates. New J. Phys. 2011, 13, 033012. [CrossRef]

16. Richaud, A.; Penna, V. Phase separation can be stronger than chaos. New J. Phys. 2018, 20, 105008. [CrossRef]

17. Lingua, F.; Richaud, A.; Penna, V. Residual entropy and critical behavior of two interacting boson species in a double well. Entropy 2018, 20, 84. [CrossRef]

18. Penna, V.; Richaud, A. The phase separation mechanism of a binary mixture in a ring trimer. Sci. Rep. 2018, 8, 10242. [CrossRef]

19. Richaud, A.; Zenesini, A.; Penna, V. The mixing-demixing phase diagram of ultracold heteronuclear mixtures in a ring trimer. Sci. Rep. 2019, 9, 6908. [CrossRef]

20. Penna, V.; Richaud, A. Spatial Phase Separation of a Binary Mixture in a Ring Trimer. J. Phys. Conf. Ser. 2019, 1206, 10242. [CrossRef]

21. Richaud, A.; Penna, V. Pathway toward the formation of supermixed states in ultracold boson mixtures loaded in ring lattices. Phys. Rev. A 2019, 100, 013609. [CrossRef]

22. Camesasca, M.; Kaufman, M.; Manas-Zloczower, I. Quantifying fluid mixing with the Shannon entropy. Macromol. Theory Simul. 2006, 15, 595-607. [CrossRef]

23. Lingua, F.; Mazzarella, G.; Penna, V. Delocalization effects, entanglement entropy and spectral collapse of boson mixtures in a double well. J. Phys. B At. Mol. Opt. Phys. 2016, 49, 205005. [CrossRef]

24. Fisher, M.P.A.; Weichman, P.B.; Grinstein, G.; Fisher, D.S. Boson localization and the superfluid-insulator transition. Phys. Rev. B 1989, 40, 546. [CrossRef] [PubMed]

25. Jaksch, D.; Bruder, C.; Cirac, J.I.; Gardiner, C.W.; Zoller, P. Cold Bosonic Atoms in Optical Lattices. Phys. Rev. Lett. 1998, 81, 3108. [CrossRef]

26. Greiner, M.; Mandel, O.; Esslinger, T.; Hänsch, T.W.; Bloch, I. Quantum phase transition from a superfluid to a Mott insulator in a gas of ultracold atoms. Nature 2002, 415, 39. [CrossRef] [PubMed]

27. Caleffi, F.; Capone, M.; Menotti, C.; Carusotto, I.; Recati, A. Quantum fluctuations beyond the Gutzwiller approximation in the Bose-Hubbard model. arXiv 2019, arXiv:1908.03470.

28. Sowiński, T.; García-March, M.Á. One-dimensional mixtures of several ultracold atoms: A review. Rep. Prog. Phys. 2019, 82, 104401. [CrossRef]

29. Amico, L.; Osterloh, A.; Cataliotti, F. Quantum Many Particle Systems in Ring-Shaped Optical Lattices. Phys. Rev. Lett. 2005, 95, 063201. [CrossRef]

30. Amico, L.; Aghamalyan, D.; Auksztol, F.; Crepaz, H.; Dumke, R.; Kwek, L.C. Superfluid qubit systems with ring shaped optical lattices. Sci. Rep. 2014, 4, 4298. [CrossRef]

31. Penna, V.; Richaud, A. Two-species boson mixture on a ring: A group-theoretic approach to the quantum dynamics of low-energy excitations. Phys. Rev. A 2017, 96, 053631. [CrossRef]

32. Spekkens, R.W.; Sipe, J.E. Spatial fragmentation of a Bose-Einstein condensate in a double-well potential. Phys. Rev. A 1999, 59, 3868-3877. [CrossRef]

33. Javanainen, J. Phonon approach to an array of traps containing Bose-Einstein condensates. Phys. Rev. A 1999, 60, 4902-4909. [CrossRef]

34. Ho, T.L.; Ciobanu, C.V. The Schrödinger Cat Family in Attractive Bose Gases. J. Low Temp. Phys. 2004, 135, 257-266. [CrossRef]

35. Ziń, P.; Chwedeńczuk, J.; Oleś, B.; Sacha, K.; Trippenbach, M. Critical fluctuations of an attractive Bose gas in a double-well potential. EPL 2008, 83, 64007.

36. Buonsante, P.; Burioni, R.; Vescovi, E.; Vezzani, A. Quantum criticality in a bosonic Josephson junction. Phys. Rev. A 2012, 85, 043625. [CrossRef] 
37. Buonsante, P.; Penna, V.; Vezzani, A. Dynamical bifurcation as a semiclassical counterpart of a quantum phase transition. Phys. Rev. A 2011, 84, 061601. [CrossRef]

38. Oelkers, N.; Links, J. Ground-state properties of the attractive one-dimensional Bose-Hubbard model. Phys. Rev. B 2007, 75, 115119. [CrossRef]

39. Sachdev, S. Quantum Phase Transitions; Cambridge University Press: Cambridge, UK, 2011.

40. Brandani, G.B.; Schor, M.; MacPhee, C.E.; Grubmüller, H.; Zachariae, U.; Marenduzzo, D. Quantifying disorder through conditional entropy: an application to fluid mixing. PLoS ONE 2013, 8, e65617. [CrossRef] [PubMed]

41. Computational Resources Provided by HPC@POLITO. Available online: http://hpc.polito.it/ (accessed on 3 January 2020).

42. Wang, W.; Penna, V.; Capogrosso-Sansone, B. Analysis and resolution of the ground-state degeneracy of the two-component Bose-Hubbard model. Phys. Rev. E 2014, 90, 022116. [CrossRef]

43. Kordas, G.; Wimberger, S.; Witthaut, D. Decay and fragmentation in an open Bose-Hubbard chain. Phys. Rev. A 2013, 87, 043618. [CrossRef]

44. Ferrini, G.; Minguzzi, A.; Hekking, F.W.J. Number squeezing, quantum fluctuations, and oscillations in mesoscopic Bose Josephson junctions. Phys. Rev. A 2008, 78, 023606. [CrossRef]

(C) 2020 by the authors. Licensee MDPI, Basel, Switzerland. This article is an open access article distributed under the terms and conditions of the Creative Commons Attribution (CC BY) license (http://creativecommons.org/licenses/by/4.0/). 